\title{
Two-fluid Taylor-Couette flow with countercurrent axial flow: Linear theory for immiscible liquids between corotating cylinders
}

\author{
Gretchen Baier and Michael D. Graham ${ }^{\text {a) }}$ \\ Department of Chemical Engineering, University of Wisconsin-Madison, Madison, Wisconsin 53706-1691
}

(Received 7 July 1999; accepted 20 October 1999)

\begin{abstract}
We computationally investigate the stability of a pair of radially stratified immiscible liquids undergoing countercurrent axial flow in the annular gap between rapidly corotating coaxial cylinders: two-fluid Taylor-Couette flow with counterflow. A simple analysis determines conditions under which a nearly cylindrical interface is maintained in the presence of counterflow (i.e., axial pressure gradients). Stability analysis reveals that for small axial Reynolds numbers, the flow is slightly stabilized against Taylor-Couette instability, consistent with results for a single phase. At axial Reynolds numbers greater than about ten, however, the flow is susceptible to a (generally nonaxisymmetric) Kelvin-Helmholtz instability, which precedes the Taylor-Couette mode. Furthermore, new results are presented for the case without axial flow. A bifurcation to vortices that corotate with their counterparts in the other phase is found. Finally, limitations of the generalized Rayleigh criterion developed in our earlier work are elucidated. In particular, we show how it fails if one of the fluid layers is very thin. (C) 2000 American Institute of Physics.
\end{abstract}

[S1070-6631(00)00602-4]

\section{INTRODUCTION}

Taylor-Couette flow is a centrifugally induced hydrodynamic instability that occurs in the flow between coaxial cylinders, when the inner cylinder is rotated faster than a critical speed. Although this flow has attracted a great deal of study since Taylor's pioneering paper in $1923,{ }^{1}$ the extension to the flow of two liquid phases in the same geometry has received relatively little attention. Here we extend our previous analysis $^{2}$ of two radially stratified immiscible fluids to include countercurrent axial flow of the two phases.

The motivation underlying this study is the development of design principles for a novel liquid-liquid extractor. Taylor-Couette flow of a single fluid has already found applications that utilize the vortex motion to increase the performance of mass transfer operations and, in many instances, a several-fold increase in filtration and reactor performance has been demonstrated. ${ }^{3-11}$ These successes suggest that the same may be expected of a two-fluid analog. Figure 1 is a schematic of a two-fluid Taylor-Couette liquid extractor. In this device, the two phases retain their individual integrity and contact each other only at a single well-defined interface. Although the interfacial area is small, the vortex motion provides an active surface for mass transfer. In contrast, standard liquid-liquid extraction processes maximize a relatively inactive surface area by dispersing one phase as small droplets in the other phase. Such dispersion based systems are often inadequate for liquid pairs that are easily emulsifiable; these arise, for example, in bioseparations that use aqueous two-phase or reverse micelle systems. ${ }^{12}$ In a companion paper experimental and theoretical results for inter- and intraphase mass transfer are presented and indicate that two-

${ }^{a)}$ Corresponding author. Electronic mail: graham@engr.wisc.edu fluid Taylor-Couette flow with countercurrent axial flow is a very promising configuration for an extraction process. ${ }^{13}$

Joseph et al. have investigated the stability of rigid rotation of two centrifugally stratified fluids between coaxial cylinders, in the absence of gravity. ${ }^{14}$ They find that a rigid interface is linearly stable when $J_{i}>1$ and is globally stable when $J_{i}>4$, where

$$
J_{i}=-\left(\rho_{1}-\rho_{2}\right) \Omega^{2} R_{i}^{3} / \gamma
$$

and $\gamma$ is the interfacial tension, $\rho_{1}$ and $\rho_{2}$ are the densities of the inner and outer fluids, respectively, $\Omega$ is the rigid body rotation rate, and $R_{i}$ is the interface position. For the conditions present in our extractor, $J_{i} \gg 4$.

Renardy and Joseph also computationally explored the linear stability of two-fluid Couette flow with only inner cylinder rotation. ${ }^{15}$ The results they report are primarily for the critical axial wave number of the one-fluid problem, which is not generally the critical wave number of the two-fluid problem. They find that two-fluid Taylor-Couette flow may be stabilized (destabilized) by placing the less (more) viscous fluid in a layer near the inner cylinder. Also, the denser fluid may be stably located at the inner cylinder when the centrifugal effect is overcome by a favorable combination of surface tension and viscosity difference.

In related experimental work, Joseph and co-workers report several modes for two-fluid flow in a horizontal Couette cell with sealed ends and a stationary outer cylinder. ${ }^{16,17}$ The fluids investigated were viscous oils (silicone, STP, SAE40, vegetable) and water, or two viscous oils. The fluid regimes observed include (1) an emulsion undergoing one fluid Taylor-Couette flow, (2) "rollers"- -axisymmetric blobs of oil attached to the inner cylinder-and vortex motion of the water phase, and (3) axially alternating bands of emulsion 


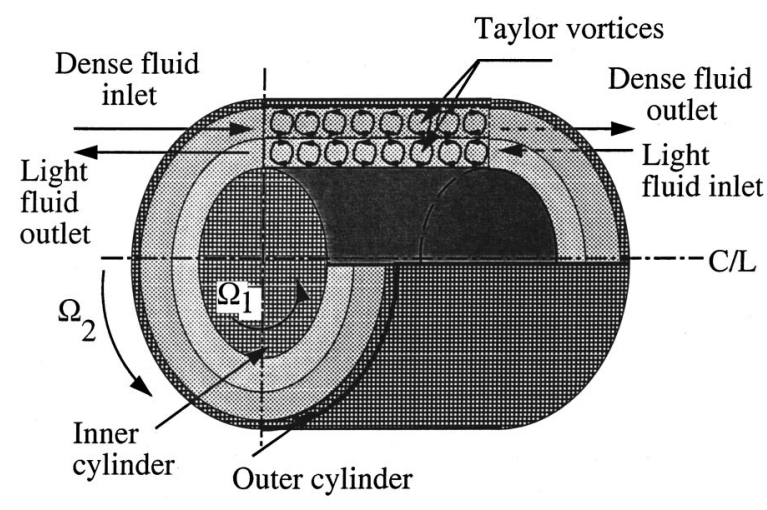

FIG. 1. Schematic of two-fluid Taylor-Couette flow with countercurrent axial flow.

and pure fluid. Campero and Vigil observed similar patterns in their experiments, which also probed axial flow effects. ${ }^{18}$

Toya and Nakamura studied Taylor-Couette flow of two fluids in a vertical annulus where the fluids are axially stratified. ${ }^{19}$ They observe the counterintuitive result that the bottom vortex in the less dense phase can co-rotate with the top vortex in the denser phase: i.e., the radial velocities have opposite signs near the boundary between the two fluids. As we describe below, an analogous result arises in the radially stratified case.

In addition to the Taylor-Couette instability in two fluid Couette flow, several studies have investigated the interfacial shear instability of fluid layers with different viscosities first predicted by Yih. ${ }^{20}$ Gallagher et $a l .{ }^{21}$ observed azimuthal waves in a vertical Couette cell filled with two radially stratified fluids of matched density, but different viscosity. Only the outer cylinder was rotated to eliminate the possibility of Taylor-Couette instability. The experimentally observed wavelength and onset of the instability agree well with the linearized theory as represented by the Orr-Sommerfeld equation for purely azimuthal viscous shear flows of two fluids.

Earlier we showed that a special case of two-fluid Taylor-Couette flow is an experimentally unobservable bifurcation of single-fluid Couette flow. ${ }^{2}$ Rayleigh's criterion ${ }^{22}$ for centrifugal instability was applied to the two-fluid case and an axisymmetric linear stability analysis for viscous fluids introduced. Because in two-fluid Couette flow the less viscous phase always displays the larger angular momentum gradient, the inviscid analysis predicts that this phase is always more susceptible to instability. Because of the interfacial boundary conditions, vortex motion will occur in both phases, but is driven by the "Rayleigh unstable" phase. A viscous analysis further showed that increasing the viscosity of the Rayleigh unstable phase is stabilizing, while increasing the viscosity of the stable phase has little effect. The density ratio also has little effect on the instability. Under experimental conditions where gravity is unimportant, the experimental and theoretical results agree quantitatively, showing that the simple inviscid analysis is useful in understanding the viscous problem. In experiments where gravity was not negligible, another instability, characterized by a longer wavelength "barber pole" pattern, was observed ex- perimentally and argued to be a two-fluid analog of rimming flow instability. ${ }^{2,23}$

The effects of axial pressure-driven flow on TaylorCouette flow of a single fluid phase have been well studied and provide a starting point for understanding the two-fluid case. Some intuition about these effects may be gained from a simple scaling argument. The centrifugal term in the radial momentum balance scales as the Taylor number $T$. The axial convection term scales as the axial Reynolds number $\mathrm{Re}_{\mathrm{ax}}$. As $\mathrm{Re}_{\mathrm{ax}}$ increases, $T$ must increase accordingly to make a leading order contribution to the radial momentum balance. Therefore, we expect the critical Taylor number to scale linearly with axial Reynolds number. This expectation is confirmed by detailed analysis and experiment. In an approximate axisymmetric analysis, Chandrasekhar found that axial flow suppresses Taylor-Couette instability and the axial wave number increases with increasing axial flow. ${ }^{24} \mathrm{Ng}$ and Turner computationally observed similar effects for axisymmetric patterns and, at very high axial Reynolds numbers, reported the dominance of a Tollmien-Schlichting shear instability in the boundary layer rather than a Taylor-Couette instability. ${ }^{25}$ Howes and Rudman showed numerically that for axisymmetric flows, some streamlines (in a reference frame traveling with the wave speed) bypass the vortex cores and flow counter to the prevailing axial flow direction; the center of each vortex travels faster than the axial mean velocity. ${ }^{26}$ Giordano et al. also observed this experimentally. ${ }^{27}$ At sufficiently high axial flowrates $\left(\mathrm{Re}_{\mathrm{ax}}\right.$ $>20$ ), the flow patterns observed experimentally and predicted theoretically are nonaxisymmetric. ${ }^{25,28}$ In a nonaxisymmetric analysis, $\mathrm{Ng}$ and Turner found that axial flow is still stabilizing and the axial wave number increases, but both effects are smaller than in the axisymmetric case. Also, nonaxisymmetric Tollmien-Schlichting instability was not observed. The nonaxisymmetric predictions of $\mathrm{Ng}$ and Turner agree well with the experimental observations of Snyder and of Donnelly and Fultz. ${ }^{29,30}$ A detailed experimental map of various flow regimes has been made by Lueptow et al.,$^{31}$ and the stabilizing effect of oscillatory axial motion of the inner cylinder has been demonstrated experimentally by Weisberg et al..$^{32}$ and computationally by Marques and Lopez. ${ }^{33}$ Incidentally, axial flow also suppresses the viscoelastic analog of Taylor-Couette instability in an inertialess Oldroyd-B model of a polymer solution; ${ }^{34,35}$ the axial stresses resist the radial deformations required for instability.

In the present work, we computationally investigate the stability of a pair of radially stratified immiscible liquids undergoing countercurrent axial flow in the annular gap between coaxial corotating cylinders. A simple analysis determines conditions under which a nearly cylindrical interface is maintained in the presence of axial pressure gradients. In the absence of axial flow, the results from the present study agree well with both our earlier work ${ }^{2}$ and with the analysis of Renardy and Joseph. ${ }^{15}$ Therefore, the assumptions of narrow gap, near rigid rotation, and axisymmetry were in fact very good for the conditions we studied previously. ${ }^{2}$ Furthermore, we find no evidence of azimuthal viscous shear instability, in agreement with the experimental observations. ${ }^{2}$ For the axial counterflow case, at small axial Reynolds numbers, 
we show that the flow is slightly stabilized against the Taylor-Couette instability, consistent with results for a single phase. At axial Reynolds numbers greater than about ten, however, the flow is susceptible to a Kelvin-Helmholtz instability, which precedes the Taylor-Couette mode.

Furthermore, new results are presented for the case without axial flow. A bifurcation to vortices that corotate with their counterparts in the other phase is found. Finally, the limitations of the generalized Rayleigh criterion developed in our earlier work are elucidated. In particular, we show how it fails if one of the fluid layers is very thin.

\section{FORMULATION}

The flow domain under consideration is the annular region between a pair of coaxial cylinders of length $L$. The inner cylinder, with radius $R_{1}$, rotates with an angular velocity of $\Omega_{1}$. Likewise, the outer cylinder, with radius $R_{2}$, rotates at $\Omega_{2}$. The aspect ratio of the device is $\Gamma=L / R_{1}$. The inner (less dense) fluid contacts only the inner cylinder and has density $\rho_{1}$, kinematic viscosity $\nu_{1}$, and dynamic viscosity $\mu_{1}=\nu_{1} \rho_{1}$. Fluid in this layer flows axially with an imposed volumetric flow rate $Q_{1}$. The base state flow field for the inner fluid is given by $\mathbf{V}_{1}=V_{1}(r) \mathbf{e}_{\boldsymbol{\theta}}+W_{1}(r) \mathbf{e}_{\mathbf{z}}$. The outer fluid is described similarly, but with subscripts 1 replaced with 2 and with the axial flow in the opposite direction. The interfacial tension between the two fluids is $\gamma$. As high rotation rates of both cylinders are required to radially stratify the two fluids, gravity is neglected in the present analysis. We assume that absent any instabilities, the interface is cylindrical, located at radial position $R_{i}$, and rotates with an angular velocity $\Omega_{i}$ that is determined from the Couette solution shown below. In the presence of axial counterflow, the interface is actually slightly tapered rather than cylindrical; we compute the extent of taper below, showing that it is negligible for the conditions of interest here. Except in the calculation of this taper, the finite length of the device is ignored and the computations performed for infinitely long cylinders.

The velocities are scaled with $\Omega_{1} R_{1}$, spatial variables with $d=R_{2}-R_{1}$, time with $d^{2} / \nu_{1}$, and pressure with $2 \Omega_{1}^{2} R_{1}^{2} \rho_{1} / \epsilon$, where $\epsilon=d / R_{2}$. The importance of interfacial tension is measured by the quantity $J=\left(\rho_{2}-\rho_{1}\right) \Omega_{2}^{2} R_{2}^{3} / \gamma$. The radial coordinate, $r$, is transformed into a new variable, $y=\left(r-R_{1}\right) / d$, which is zero at the inner cylinder and one at the outer. The position of the interface is $y_{i}$.
The azimuthal component of the base flow is two-fluid Couette flow with boundary conditions of no-slip at the cylinder walls, and continuity of shear stress and velocity at the interface. The resulting Couette solution has only an azimuthal velocity component for each phase $(j=1,2)$ :

$$
V_{j}=A_{j}\left(y+\frac{R_{12}}{\epsilon}\right)+\frac{B_{j}}{\left(y+R_{12} / \epsilon\right)}
$$

where

$$
\begin{aligned}
A_{1} & =\frac{\epsilon}{R_{12}}-\frac{\mu_{21}\left(\Omega_{21}-1\right) \epsilon / R_{12}}{R_{12}^{2}-\mu_{21}+R_{1 i}^{2}\left(\mu_{21}-1\right)} \\
& =\frac{\left(R_{1 i}^{2}-\Omega_{i 1}\right) \epsilon / R_{12}}{R_{1 i}^{2}-1}, \\
B_{1} & =\frac{\mu_{21}\left(\Omega_{21}-1\right) R_{12} / \epsilon}{R_{12}^{2}-\mu_{21}+\left(\mu_{21}-1\right) R_{1 i}^{2}}=\frac{\left(\Omega_{i 1}-1\right) R_{12} / \epsilon}{R_{1 i}-1}, \\
A_{2} & =\Omega_{21} \frac{\epsilon}{R_{12}}-\frac{\left(\Omega_{21}-1\right) \epsilon / R_{12}}{1-\mu_{21} R_{21}^{2}+R_{2 i}^{2}\left(\mu_{21}-1\right)} \\
& =\frac{\Omega_{i 1}\left(R_{i 2}^{2}-\Omega_{2 i}\right) \epsilon / R_{12}}{R_{i 2}-1}, \\
B_{2} & =\frac{1}{\mu_{21}} B_{1}=\frac{\Omega_{i 1} R_{i 1}^{2}\left(\Omega_{2 i}-1\right) R_{12} / \epsilon}{R_{i 2}-1} .
\end{aligned}
$$

Here $R_{1 i}=R_{1} / R_{i}, \Omega_{21}=\Omega_{2} / \Omega_{1}, \mu_{21}=\mu_{2} / \mu_{1}$, etc., and $\Omega_{i}$ is determined from Eq. (1).

Similarly, the base axial velocity for each phase is

$$
W_{j}=F_{j}\left(y+\frac{R_{12}}{\epsilon}\right)^{2}+G_{j} \ln \left(y+\frac{R_{12}}{\epsilon}\right)+H_{j},
$$

where

$$
\begin{aligned}
& F_{1}=\frac{\Delta P_{z, 1}}{4 \Gamma \Omega_{21} \mathrm{Ek}_{1} \epsilon}, \quad F_{2}=\frac{\Delta P_{z, 2}}{4 \Gamma \Omega_{21} \mathrm{Ek}_{2} \epsilon \mu_{21}}, \\
& G_{1}=-2\left(\frac{R_{12}}{\epsilon}+y_{i}\right)^{2}\left(F_{1}-\mu_{21} F_{2}\right)+\mu_{21} G_{2}, \\
& H_{1}=-F_{1}\left(\frac{R_{12}}{\epsilon}\right)^{2}-G_{1} \ln \left(\frac{R_{12}}{\epsilon}\right),
\end{aligned}
$$

$G_{2}=-\frac{\left[\left(F_{1}-F_{2}\right)\left(R_{12} / \epsilon+y_{i}\right)^{2}-F_{1}\left(R_{12} / \epsilon\right)^{2}+F_{2}\left(1+R_{12} / \epsilon\right)^{2}-2\left(F_{1}-\mu_{21} F_{2}\right)\left(R_{12} / \epsilon+y_{i}\right)^{2}\left[\ln \left(R_{12} / \epsilon+y_{i}\right)-\ln \left(R_{12} / \epsilon\right)\right]\right]}{\ln \left(1+R_{12} / \epsilon\right)+\left(\mu_{21}-1\right) \ln \left(R_{12} / \epsilon+y_{i}\right)-\mu_{21} \ln \left(R_{12} / \epsilon\right)}$,

$$
H_{2}=-F_{2}\left(1+\frac{R_{12}}{\epsilon}\right)^{2}-G_{2} \ln \left(1+\frac{R_{12}}{\epsilon}\right) \text {. }
$$

$$
\mathrm{Ek}_{j}=\frac{\mu_{j}}{2 \rho_{j} \Omega_{2} d^{2}}
$$




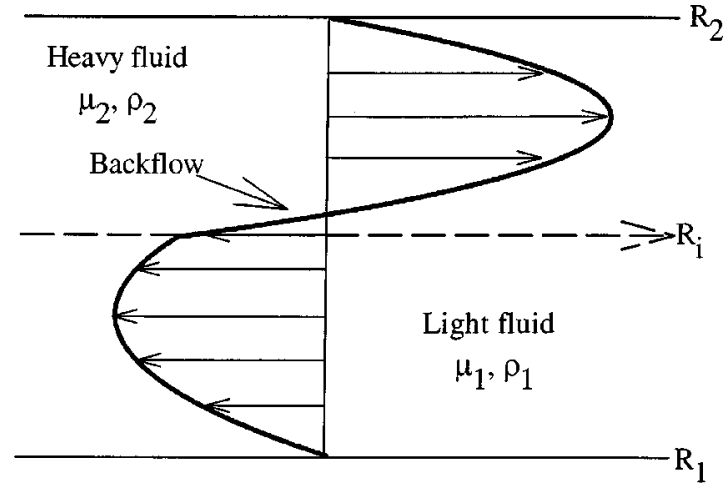

FIG. 2. Velocity profile for the countercurrent axial base flow. Here $R_{12}$ $=0.826, Q_{21}=1, \mu_{21}=0.22$, and $y_{i}=0.5$.

by the volumetric flow rates. The flow rate ratio $Q_{21}$ is the (absolute value of the) ratio between the volumetric flow rates of the outer and inner phases.

Since the axial counterflow is driven by the axial pressure drop for the respective phase, the interface is slightly tapered rather than cylindrical, as was assumed in the above solutions for the base velocities. A perturbation analysis of the normal stress boundary condition, neglecting surface tension, reveals that at rigid rotation in the narrow gap limit:

$\left.\left(P_{1}-P_{2}\right)\right|_{y=y_{i}}=\left(\frac{\left(\rho_{2}-\rho_{1}\right) \Omega_{2}^{2} R_{2} \delta}{L}+\left(\frac{\partial P_{2}}{\partial z}-\frac{\partial P_{1}}{\partial z}\right)\right) z=0$,

where $\delta+R_{i}$ is the maximum radius of the tapered interface. The maximum taper is then

$$
\frac{\delta}{d}=\frac{4 \Gamma R_{12} \operatorname{Re}_{\mathrm{ax}, 2} E k_{2}^{2}}{\left(1-\rho_{12}\right)}\left(\mu_{12}^{2} \rho_{21} \frac{\operatorname{Re}_{\mathrm{ax}, 1}}{\operatorname{Re}_{\mathrm{ax}, 2}}+1\right),
$$

where $\operatorname{Re}_{\mathrm{ax}, \mathrm{j}}=\left|\overline{W_{j}}\right| d / \nu_{j}$ and $\overline{W_{j}}$ is the radially averaged base axial velocity. The taper increases with increasing axial flow or an increasing difference in the flow rates, but decreases with increasing centrifugal force or increasing density difference. When $\delta$ is small, the above solutions using a cylindrical interface at $y_{i}$ are good approximations. For the results presented in this paper, $\delta / d<0.04$ with $\Gamma=6$.

A typical axial velocity profile for countercurrent axial flow is shown in Fig. 2. In general the axial velocity at the interface is nonzero and the result is backflow in one of the fluid phases. Furthermore, Rayleigh's theorem for inviscid flow profiles with inflection points suggests that the flow might become unstable at sufficiently high axial Reynolds numbers. $^{22}$

\section{A. Stability analysis}

We now consider the evolution of perturbations to the base flow; the velocity field is $\mathbf{v}_{j}=\mathbf{V}_{j}+\hat{\mathbf{v}}_{j}, j=1,2$. The disturbance flow is assumed to have the following normal mode form:

$$
\hat{\mathbf{v}}_{j}=\left(\begin{array}{c}
\mathbf{v}_{r, j}(y) \\
\mathbf{v}_{\theta, j}(y) \\
\mathbf{v}_{z, j}(y)
\end{array}\right) e^{i \alpha(z-c t)+i n \theta+c . c .}
$$

The interface deforms from the flat unperturbed interface at $y_{i}$ as $\hat{h}=h e^{i \alpha(z-c t)+i n \theta}+$ c.c. Pressure also has the form $\hat{p}$ $=p(y) e^{i \alpha(z-c t)+i n \theta}+\mathrm{c}$. c. The governing equations and boundary conditions for the disturbance quantities are linearized and domain perturbation is used to apply the interfacial boundary conditions at the unperturbed interface position, $y_{i}$, rather than at the actual unknown interface position.

The linearized governing equations are radial momentum:

$$
\begin{gathered}
{\left[-i \alpha c-\mu_{j 1} \rho_{1 j}\left(\frac{\partial^{2}}{\partial y^{2}}+\frac{1}{\left(y+R_{12} / \epsilon\right)} \frac{\partial}{\partial y}\right.\right.} \\
\left.-\frac{\left(1+n^{2}\right)}{\left(y+R_{12} / \epsilon\right)^{2}}-\alpha^{2}\right)+i n \frac{R_{12}}{2 \mathrm{Ek}_{1} \Omega_{21} \epsilon} \frac{V_{j}}{\left(y+R_{12} / \epsilon\right)} \\
\left.+i \alpha \frac{R_{12}}{2 \mathrm{Ek}_{1} \Omega_{21} \epsilon} W_{j}\right] \mathrm{v}_{r, j}+\left[-2 \frac{R_{12}}{2 \mathrm{Ek}_{1} \Omega_{21} \epsilon} \frac{V_{j}}{\left(y+R_{12} / \epsilon\right)}\right. \\
\left.+\mu_{j 1} \rho_{1 j} \frac{2 i n}{\left(y+R_{12} / \epsilon\right)^{2}}\right] \mathrm{v}_{\theta, j}+\rho_{1 j} \frac{2}{\epsilon} \frac{R_{12}}{2 \mathrm{Ek}_{1} \Omega_{21} \epsilon} \frac{\partial p_{j}}{\partial y}=0,
\end{gathered}
$$

azimuthal momentum:

$$
\begin{aligned}
& {\left[-i \alpha c-\mu_{j 1} \rho_{1 j}\left(\frac{\partial^{2}}{\partial y^{2}}+\frac{1}{\left(y+R_{12} / \epsilon\right)} \frac{\partial}{\partial y}\right.\right.} \\
& \left.-\frac{\left(1+n^{2}\right)}{\left(y+R_{12} / \epsilon\right)^{2}}-\alpha^{2}\right)+i n \frac{R_{12}}{2 E k_{1} \Omega_{21} \epsilon} \frac{V_{j}}{\left(y+R_{12} / \epsilon\right)} \\
& \left.+i \alpha \frac{R_{12}}{2 \mathrm{Ek}_{1} \Omega_{21} \epsilon} W_{j}\right] \mathrm{v}_{\theta, j}+\left[2 A_{j} \frac{R_{12}}{2 E k_{1} \Omega_{21} \epsilon}\right. \\
& \left.-\mu_{j 1} \rho_{1 j} \frac{2 i n}{\left(y+R_{12} / \epsilon\right)^{2}}\right] \mathrm{v}_{r, j} \\
& +i n \rho_{1 j} \frac{2}{\epsilon\left(y+R_{12} / \epsilon\right)} \frac{R_{12}}{2 \mathrm{Ek}_{1} \Omega_{21} \epsilon} p_{j}=0
\end{aligned}
$$

axial momentum:

$$
\begin{gathered}
{\left[-i \alpha c-\mu_{j 1} \rho_{1 j}\left(\frac{\partial^{2}}{\partial y^{2}}+\frac{1}{\left(y+R_{12} / \epsilon\right)} \frac{\partial}{\partial y}-\frac{n^{2}}{\left(y+R_{12} / \epsilon\right)^{2}}-\alpha^{2}\right)\right.} \\
\left.+i n \frac{R_{12}}{2 \mathrm{Ek}_{1} \Omega_{21} \epsilon} \frac{V_{j}}{\left(y+R_{12} / \epsilon\right)}+i \alpha \frac{R_{12}}{2 \mathrm{Ek}_{1} \Omega_{21} \epsilon} W_{j}\right] \mathrm{v}_{z, j} \\
+\frac{R_{12}}{2 \mathrm{Ek}_{1} \Omega_{21} \epsilon} \frac{\partial W_{j}}{\partial y} \mathrm{v}_{r, j}+i \alpha \rho_{1 j} \frac{2}{\epsilon} \frac{R_{12}}{2 \mathrm{Ek}_{1} \Omega_{21} \epsilon} p_{j}=0,
\end{gathered}
$$

continuity:

$\frac{\mathrm{v}_{r, j}}{\left(y+R_{12} / \epsilon\right)}+\frac{\partial \mathrm{v}_{r, j}}{\partial y}+\frac{i n}{\left(y+R_{12} / \epsilon\right)} \mathrm{v}_{\theta, j}+i \alpha \mathrm{v}_{z, j}=0$.

The linearized interfacial boundary conditions require that at $y=y_{i}$,

$\mathrm{v}_{r, 1}=\mathbf{v}_{r, 2}$, 


$$
\begin{aligned}
& \mathrm{v}_{\theta, 1}-\mathrm{v}_{\theta, 2}-h\left[\frac{2\left(B_{1}-B_{2}\right)}{\left(y+R_{12} / \epsilon\right)^{2}}\right]=0, \\
& \mathrm{v}_{z, 1}-\mathrm{v}_{z, 2}+h\left[2\left(y+\frac{R_{12}}{\epsilon}\right)\left(F_{1}-F_{2}\right)+\frac{\left(G_{1}-G_{2}\right)}{\left(y+R_{12} / \epsilon\right)}\right]=0, \\
& \frac{\partial \mathrm{v}_{z, 1}}{\partial y}-\mu_{21} \frac{\partial \mathrm{v}_{z, 1}}{\partial y}+i \alpha\left(1-\mu_{21}\right) \mathrm{v}_{r, 2} \\
& \quad+h\left[2\left(F_{1}-\mu_{21} F_{2}\right)-\frac{\left(G_{1}-\mu_{21} G_{2}\right)}{\left(y+R_{12} / \epsilon\right)^{2}}\right]=0, \\
& \frac{\partial \mathrm{v}_{\theta, 1}}{\partial y}-\frac{\mathrm{v}_{\theta, 1}}{\left(y+R_{12} / \epsilon\right)}-\mu_{21} \frac{\partial \mathrm{v}_{\theta, 2}}{\partial y}+\mu_{21} \frac{\mathrm{v}_{\theta, 2}}{\left(y+R_{12} / \epsilon\right)} \\
& \quad+\frac{i n\left(1-\mu_{21}\right)}{\left(y+R_{12} / \epsilon\right)} \mathrm{v}_{r, 2}=0, \\
& \frac{1}{1-\rho_{21}}\left[\left(p_{1}-p_{2}\right)(1-\epsilon)-2 \Omega_{21} \mathrm{Ek}_{1} \epsilon^{2}\left(\frac{\partial \mathrm{v}_{r, 1}}{\partial y}-\mu_{21} \frac{\partial \mathrm{v}_{r, 2}}{\partial y}\right)\right] \\
& \quad+h\left[\frac{d}{2 R_{i}}(1-\epsilon) \epsilon V_{2}^{2}-\frac{\Omega_{21}}{4 \mathrm{Ek}_{1} J \epsilon}\left(\alpha^{2}-\left(\frac{d}{R_{i}}\right)^{2}\left(1-n^{2}\right)\right)\right] \\
& =0,
\end{aligned}
$$

with the linearized kinematic condition

$$
h\left(\frac{-i \alpha c}{\operatorname{Re}_{1}}+\frac{d}{R_{i}} i n V_{2}+i \alpha W_{2}\right)=\mathrm{v}_{r, 2} .
$$

No-slip conditions apply at $y=0$ and $y=1$. The equations were discretized using Chebyshev collocation: ChebyshevGauss-Lobatto integration points were used for the velocities and Chebyshev-Gauss points for pressure. ${ }^{36}$ Results here were performed with 16 collocation points in each phase; this was found to be more than sufficient for convergence. The resulting algebraic generalized eigenvalue problem is solved with a public-domain subroutine that uses QZ factorization. ${ }^{37}$ Instability occurs when $\operatorname{Im}(c)$ becomes positive.

\section{RESULTS}

\section{A. Two-fluid Taylor-Couette flow without axial countercurrent flow}

We begin by reviewing and extending our earlier results ${ }^{2}$ in the absence of axial flow. Without countercurrent flow, a simple stability criterion can be derived by considering each layer to be inviscid but with the viscous Couette flow velocity profile. Each phase is then subject to a Rayleigh criterion similar to that for the one fluid case, ${ }^{22,2}$ where instability occurs when $\Omega_{21}<R_{12}^{2}$. We find that instability occurs in the inner fluid when

$$
\Omega_{21}<\Omega_{21, c, 1}=\frac{R_{12}^{2}+\left(\mu_{21}-1\right) R_{1 i}^{2}}{\mu_{21}}
$$

and in the outer fluid when

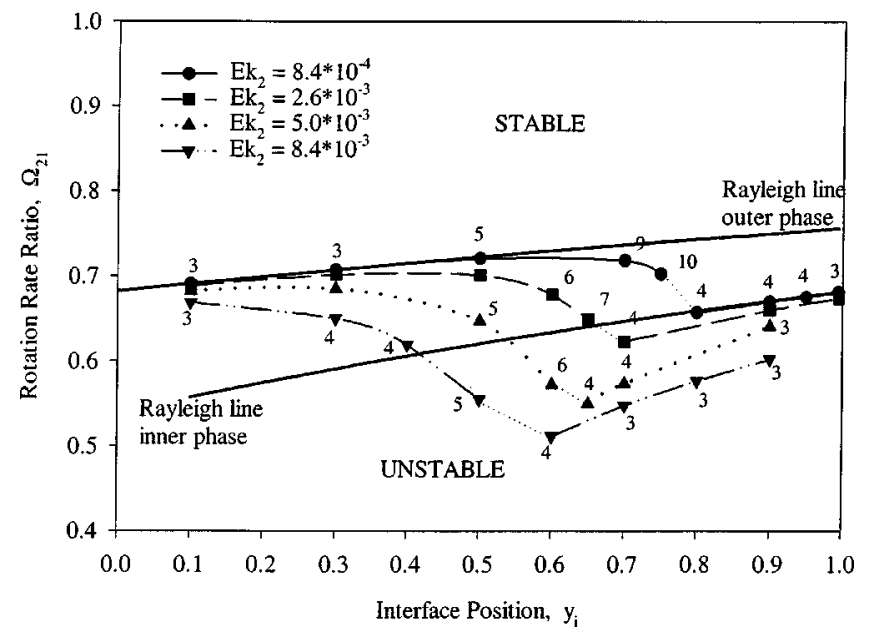

FIG. 3. The effect of interface position on the critical rotation rate ratio. Here $\mu_{21}=0.69, \rho_{21}=1.35 ; \operatorname{Re}_{\mathrm{ax}, 1}=\operatorname{Re}_{\mathrm{ax}, 2}=0$. The numbers represent the critical axial wave number, $\alpha_{c}$, to the nearest integer.

$$
\Omega_{21}<\Omega_{21, c, 2}=\frac{R_{12}^{2}}{\mu_{21}-\left(\mu_{21}-1\right) R_{1 i}^{2}} .
$$

These criteria predict that independent of interface position, the less viscous fluid is always the one more susceptible to instability in inviscid limit. Based on the Rayleigh criterion [Eqs. (4) and (5)], a phase becomes unstable when its Taylor number becomes positive, as can be seen from the bracketed terms in the definitions of the Taylor numbers for the two phases:

$$
T_{1}=\frac{\left[R_{1 i}^{2}-\Omega_{i 1}\right]}{\left(1-R_{1 i}^{2}\right)} \frac{\Omega_{12}^{2}}{\mathrm{Ek}_{1}^{2}}
$$

and

$$
T_{2}=\frac{\left[R_{i 2}^{2}-\Omega_{2 i}\right]}{\left(1-R_{i 2}^{2}\right)} \frac{\Omega_{i 2}^{2}}{\mathrm{Ek}_{2}^{2}} .
$$

It should be noted that for a given geometry and pair of fluids, the two Taylor numbers are not independent. Furthermore, since $\Omega_{21}$ is the relevant experimentally tunable parameter and is the natural bifurcation parameter of the inviscid analysis, it is used to represent most of our results.

When the fluids are of equal depth, a full viscous analysis predicts no qualitative differences from the inviscid analysis. ${ }^{2}$ However, when the less viscous fluid layer is sufficiently thin (i.e., where $y_{i}$ or $1-y_{i}$ is small), viscous effects become important and can cause the more viscous layer to be the less stable phase, contradicting the predictions of the inviscid theory. Figure 3 shows the effect of interface position for $\mu_{21}=0.69$ and several Ekman numbers. Unless otherwise specified, the results are for operating conditions representative of our current experimental apparatus: ${ }^{13} R_{2}=5.05 \mathrm{~cm}$, $R_{12}=0.826, \quad y_{i}=0.5, \quad \Omega_{2}=8 \mathrm{rev} / \mathrm{sec}, \quad \mu_{2}=7.52 \mathrm{cp}, \quad \rho_{2}$ $=1.15 \mathrm{~g} / \mathrm{ml}, 0.22<\mu_{21}<4.85,1.20<\rho_{21}<1.5$, and $\gamma \approx 50$ dyne/cm, giving $J=1939$. The Rayleigh criteria for the inner and outer fluids and the critical axial wave number, $\alpha_{c}$, are also shown. From this figure, it is observed that (1) increas- 


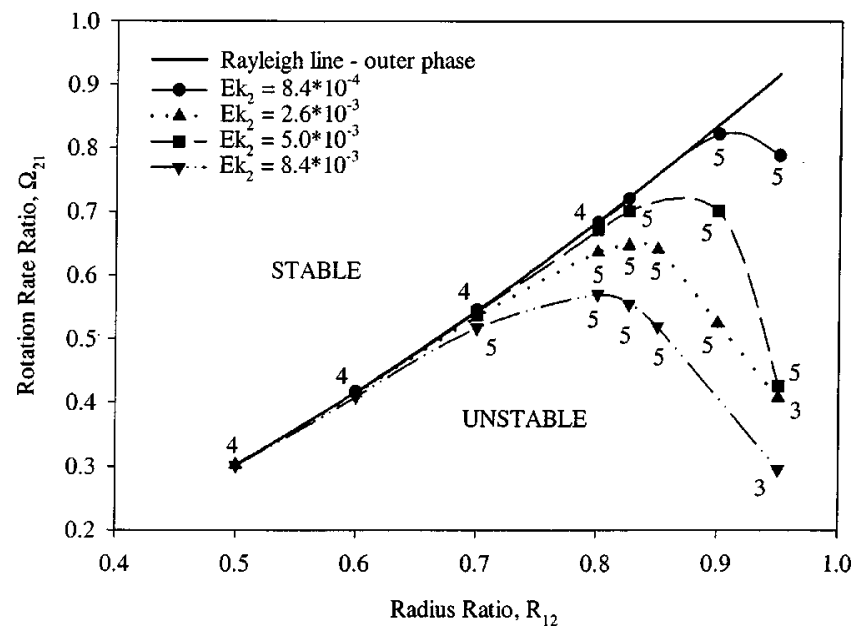

FIG. 4. The effect of radius ratio on the critical rotation rate ratio. Here $\mu_{21}=0.69, \rho_{21}=1.35 ; \operatorname{Re}_{\mathrm{ax}, 1}=\operatorname{Re}_{\mathrm{ax}, 2}=0$. The numbers represent the critical axial wave number, $\alpha_{c}$, to the nearest integer.

ing the Ekman number is stabilizing, as found in our earlier work, ${ }^{2}$ and (2) the less viscous phase is the unstable phase until a critical thinness of that layer. The vortices remain approximately square and the axial wave number indicates which phase is unstable. The abrupt change in wave number identifies the critical thinness where the switch occurs from one phase being the unstable phase to the other. Interestingly, after the switch the stability is given to a good approximation by the Rayleigh criterion for the thicker, more viscous layer. As the effective viscosity (Ekman number) increases, the critical thinness required approaches $y_{i}=0.5$.

As the radius ratio approaches unity, the Rayleigh criterion predicts that the rotation rate ratio for instability also approaches unity. However, viscous effects again become more important as the layers become thinner. The flow is stabilized as the Ekman number increases, which occurs when the viscosity increases, the rotation rate $\left(\Omega_{2}\right)$ decreases or the gap width decreases $\left(R_{12} \rightarrow 1\right)$. Figure 4 shows the stabilizing effects of narrowing the gap on increasing Ekman number.

In addition to the stabilizing effects of Ekman number, increasing surface tension slightly stabilizes the system. Surface tension has a greater effect at higher Ekman numbers, although the effect remains small; for $\mathrm{Ek}_{2}=8.4 \times 10^{-3}$, $\Omega_{21} / \Omega_{21, J=\infty} \rightarrow 0.982$ as $J \rightarrow 0$. The dimensionless group measuring the surface tension term in the normal stress boundary condition is small for the conditions studied here $\left[\Omega_{21} / 4 J \mathrm{Ek}_{1} \epsilon\left(1-\rho_{21}\right)<1\right]$.

For all of the cases studied, when the Taylor-Couette instability first appears, vortices paired across the interface counterrotate; i.e., points just across the interface from one another have very similar velocities. However, at finite amplitude, two commercial computational fluid dynamics codes (FLUENT and FIDAP, Fluent, Inc., Lebanon, NH) predict that the vortex pattern can change to a stable corotation state; i.e., the sign of the axial velocity (relative to the velocity of the interface) changes across the interface. ${ }^{13}$ Recall that Toya and Nakamura ${ }^{19}$ observed a similar behavior in axially stratified two-fluid Couette flow. Since our linear stability analysis is valid only at the onset of two-fluid Taylor-Couette flow, it cannot directly determine supercritical flow behavior. However, the second mode to go unstable in the linear stability analysis can give an indication of other important flow patterns. When the two fluids are identical, with equal fluid depths, negligible curvature, and no countercurrent axial flow, the linear stability analysis predicts counterrotating vortices as the primary unstable mode. A corotating vortex state is the second mode to bifurcate and may become stable at finite amplitude due to mode interactions. ${ }^{38}$ However, this mode is significantly affected by counterflow, as shown for $Q_{21}=1$ in Fig. 5. Once Re $>3$, the first and second modes look similar, the difference being which phase has stronger vortices. This suggests that countercurrent axial flow sufficiently affects the problem symmetry that it eliminates corotating vortices. From the mass transfer point of view, this result is fortunate, as the interphase mass transfer rate is expected to be higher $\left[O\left(\mathrm{Pe}^{1 / 2}\right)\right.$, for Péclet number Pe $\left.\gg 1\right]$ between counterrotating vortices than between corotating ones $\left[O\left(\mathrm{Pe}^{1 / 3}\right)\right]$. The difference arises because in the latter case, there is, roughly speaking, an effective no-slip condition at the interface, so convective transport is less effective. ${ }^{39}$

\section{B. Two-fluid Taylor-Couette flow with counterflow}

The main effects of countercurrent axial flow are summarized in Figs. 6 and 7. For comparison the single fluid results of $\mathrm{Ng}$ and Turner $^{25}$ are also shown. From these graphs the following observations may be made: (1) At low flow rates there is no effect on the onset of the vortex flow or on the structure of the vortices as described by the axial and azimuthal wave numbers. (2) At moderate flow rates the flow is slightly stabilized and both wave numbers increase; the vortices become thinner and helical. These results agree qualitatively with similar observations for one-fluid TaylorCouette flow. ${ }^{24,25,28}$ For both the one- and two-fluid cases the relation $T_{c} \propto \mathrm{Re}_{\mathrm{ax}}$ holds for the axisymmetric case at moderate flow rates, in agreement with the scaling argument presented in the Introduction. (3) At higher flow rates, the flow is strongly destabilized, particularly with respect to nonaxisymmetric disturbances, indicating the appearance of another instability. In the two-fluid problem, this instability is a Kelvin-Helmholtz mode, as we discuss below, and appears when $\mathrm{Re}_{\mathrm{ax}, 2} \approx 10-100$. At sufficiently high $\mathrm{Re}_{\mathrm{ax}}$, this instability will occur even at rigid rotation. In contrast, the single fluid problem is stable to much higher axial Reynolds numbers $(\approx 7000)$, where a Tollmien-Schlichting mode apparently appears. ${ }^{25}$

Several disturbance stream functions for axisymmetric flow with $Q_{21}=1$ and $\mu_{21}=0.69$ are illustrated in Fig. 8, in the reference frame traveling with the wave speed $\operatorname{Re}(c)$. The stream functions shown are a sum of the disturbance and base state stream functions, with the amplitude of the disturbance velocities chosen to be comparable with the base state axial velocities. The direction of wave propagation generally corresponds to the flow direction in the fluid where the vortices are stronger. The vortices in both phases travel in that same direction, although the flow overall is countercurrent. 
Eirst unstable mode

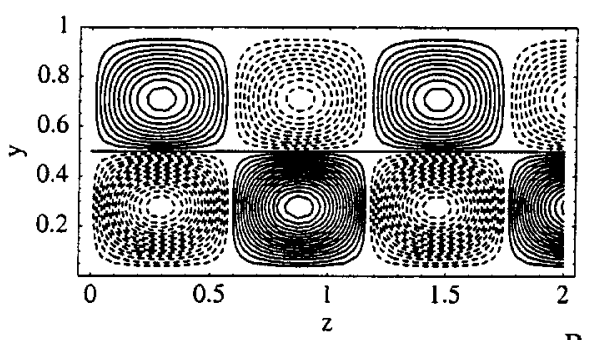

(a)

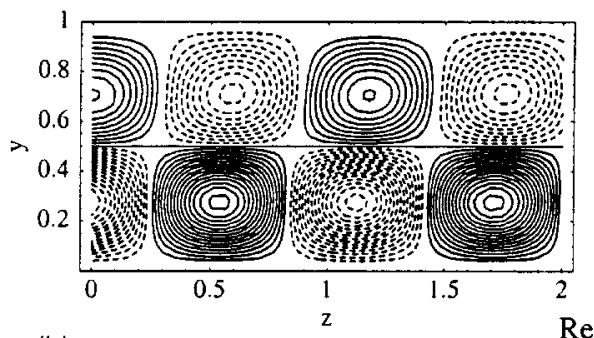

(b)

$$
\operatorname{Re}_{a x, 2}=0.33
$$
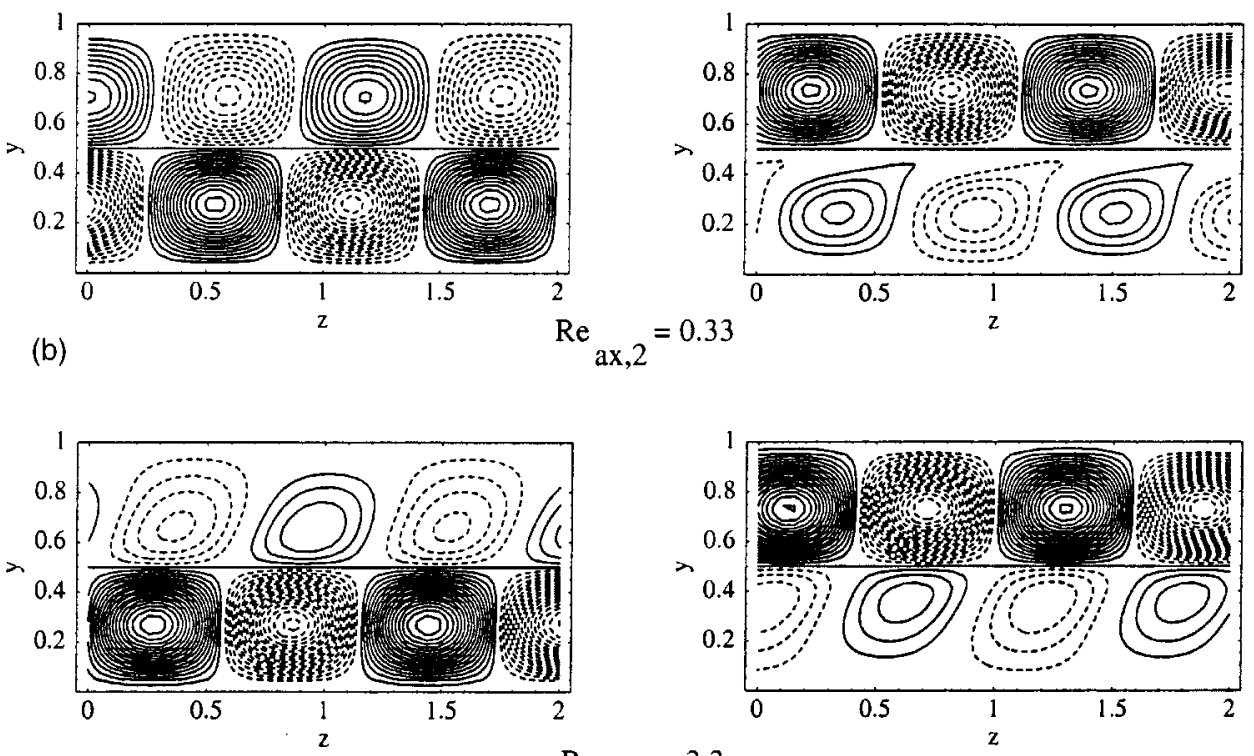

(c)

$$
\operatorname{Re}_{\mathrm{ax}, 2}=3.3
$$

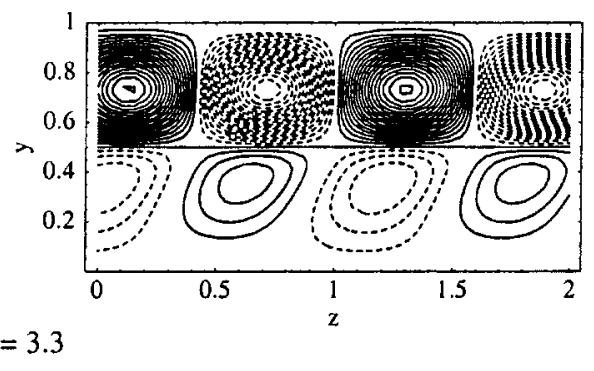

Second unstable mode

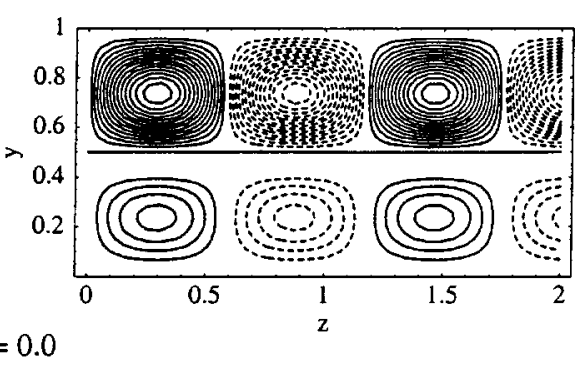

FIG. 5. The stream function, in a reference frame traveling with the wave speed, $\operatorname{Re}(c)$, for the first and second unstable modes (as $\Omega_{21}$ decreases) with increasing countercurrent axial flow. Here $\alpha=5.365, n=0, \mathrm{Ek}_{2}=8.4$ $\times 10^{-4}, \quad \mu_{21}=1, \quad \rho_{21}=1.0145, \quad Q_{21}$ $=1$, and $\Omega_{21, c}=0.679$. Positive and negative values are solid and dashed, respectively.
As seen in the figures, some streamlines "bypass" the vortex structure, and a characteristic pattern of stagnation points develops. With increasing axial Reynolds numbers, the vortices become thinner and one vortex appears to be drawn under its neighbor. At higher Reynolds numbers, a com-

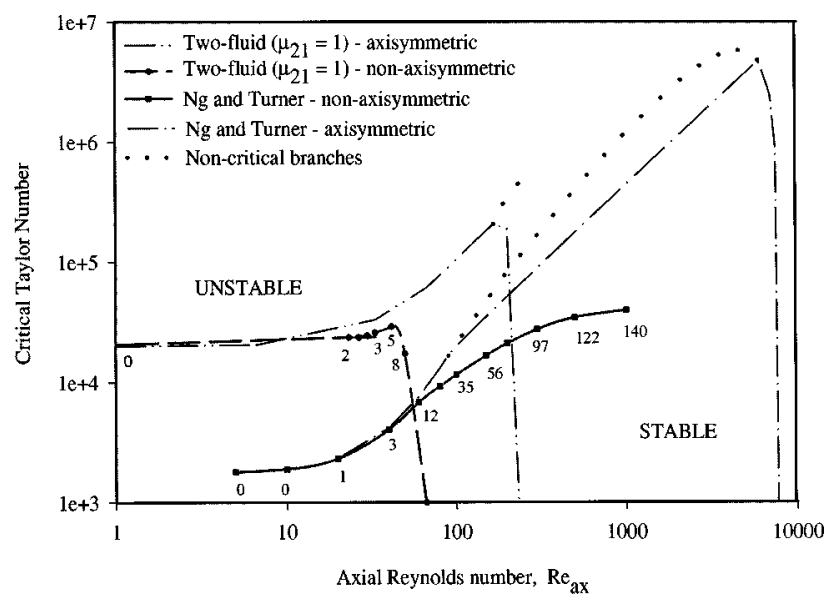

FIG. 6. The critical Taylor number for one-fluid Taylor-Couette flow with axial flow and two-fluid Taylor-Couette flow with countercurrent axial flow. For the two-fluid case $\rho_{21}=1.35, Q_{21}=1$, and $\mu_{21}=1.0$. For the nonaxisymmetric cases, the numbers represent the critical azimuthal wave number, $n_{c}$. Ng and Turner's one fluid results are for $R_{12}=0.95, \Omega_{2}=0$. pletely different pattern appears corresponding to the strongly destabilizing mode mentioned above. The stagnation point pattern shifts to that characteristic of the Kelvin's

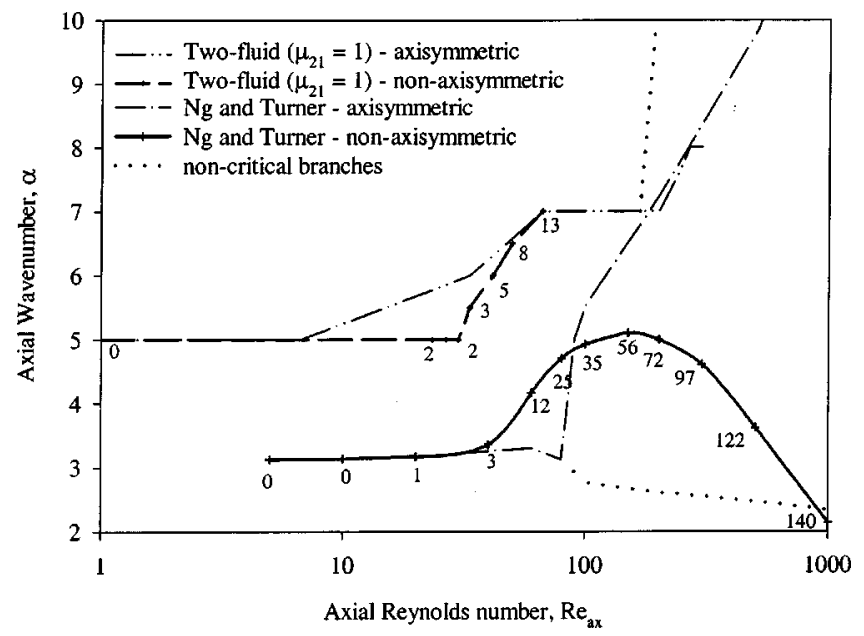

FIG. 7. The critical axial wave number for one-fluid Taylor-Couette flow with axial flow and two-fluid Taylor-Couette flow with countercurrent axial flow. For the two fluid case $\rho_{21}=1.35, Q_{21}=1$, and $\mu_{21}=1.0$. For the nonaxisymmetric cases, the numbers represent the azimuthal wave number, $n_{c}$. $\mathrm{Ng}$ and Turner's one fluid results are for $R_{12}=0.95, \Omega_{2}=0$. 


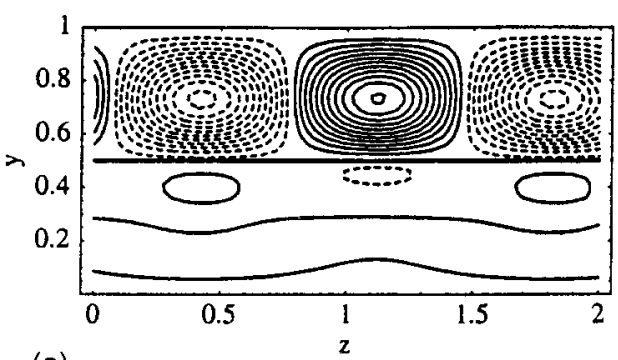

(a)
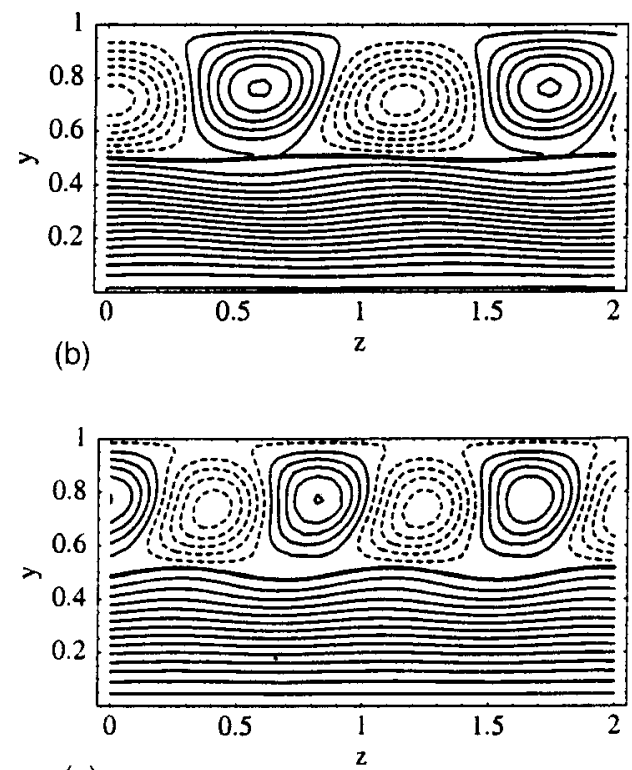

(c)

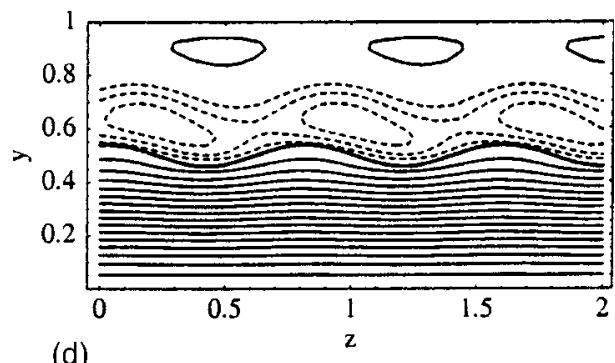

(d)

FIG. 8. Stream functions, in a reference frame traveling with the wave speed, $\operatorname{Re}(c)$, for axisymmetric two-fluid Taylor-Couette flow with countercurrent axial flow. Positive and negative values are solid and dashed, respectively. The dark line is the interface. Here $\rho_{21}=1.35, \mathrm{Ek}_{2}=8.4 \times 10^{-4}, n$ $=0, Q_{21}=1$, and $\mu_{21}=0.69$. (a) $\operatorname{Re}_{\mathrm{ax}, 2}=0.33, \quad \alpha=4.5, \quad \Omega_{21}=\Omega_{21 c}$ $=0.7206$, (b) $\operatorname{Re}_{\mathrm{ax}, 2}=33, \alpha=5.5, \Omega_{21}=\Omega_{21 c}=0.7181$, (c) $\operatorname{Re}_{\mathrm{ax}, 2}=100, \alpha$ $=7.5, \quad \Omega_{21}=\Omega_{21 c}=0.7081, \quad$ and $(\mathrm{d}) \quad \operatorname{Re}_{\mathrm{ax}, 2}=232, \quad \alpha=8, \quad \Omega_{21}=\Omega_{21 c}$ $=0.7335$.

"cat's eye" pattern of the Kelvin-Helmholtz instability.

In general, the first derivative of the base state axial flow is discontinuous at the interface and the flow profile does not have an inflection point elsewhere. However, the change in concavity may be measured by the jump in the second derivative $\left.\left(d^{2} W_{1} / d y^{2}-d^{2} W_{2} / d y^{2}\right)\right|_{y=y_{i}}$. Alternately, this quantity measures how sharply peaked the vorticity is. As $Q_{21}$ decreases with $\mu_{21}$ fixed, or as $\mu_{21}$ increases with $Q_{21}$ fixed, this jump increases and the flow is expected to be destabilized, consistent with Rayleigh's and Fjortoft's theorems. ${ }^{22}$ In agreement with this argument, Fig. 9 shows

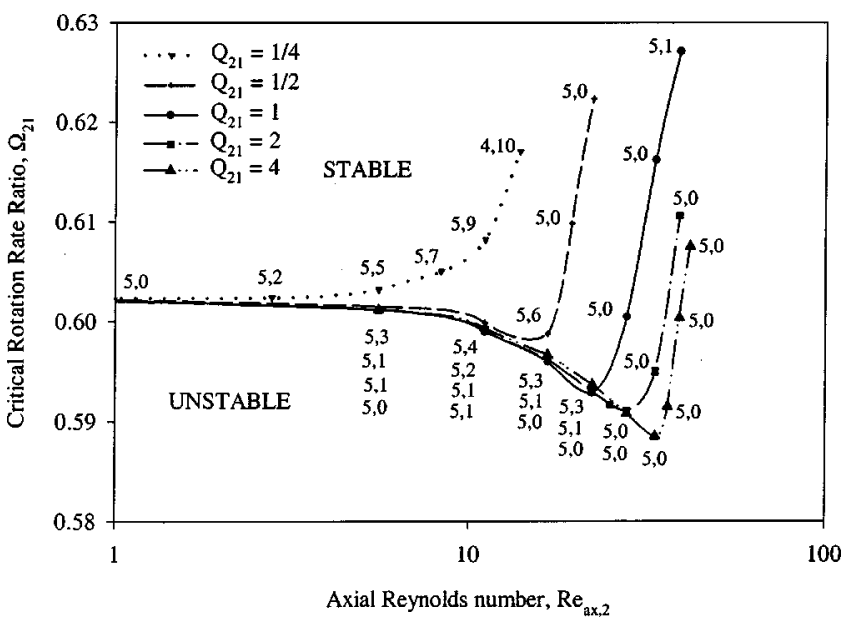

FIG. 9. The effect of unmatched countercurrent flowrates. Here $\mu_{21}=1.0$, $\rho_{21}=1.35, \mathrm{Ek}_{2}=5.04 \times 10^{-3}$, and $\Omega_{21 \mathrm{Re}_{\mathrm{ax}, 2}=0}=0.6024$. The numbers represent the critical axial and azimuthal wave numbers $\alpha_{c}$ and $n_{c}$ to the nearest integer.

the destabilizing effect of decreasing $Q_{21}$ for matched viscosity fluids $\left(\mu_{21}=1\right)$ and Fig. 10 shows the same effect with increasing $\mu_{21}$ for matched flow rates $\left(Q_{21}=1\right)$. We note that, as axial Reynolds number increases, the neutral curves become extremely flat, and it becomes difficult to accurately determine the critical wave numbers. This difficulty is reflected in the figures, where significant variations in the critical azimuthal wave number can be seen.

The effect of Ekman number is shown in Figs. 11 and 12. Note the normalization of $\Omega_{21}$ with the values for zero counterflow, $\Omega_{21, \operatorname{Re}_{\mathrm{ax}, 2}=0}$. With increasing viscosity, the second instability occurs at a higher average axial velocity, but at a lower axial Reynolds number (the average axial velocity

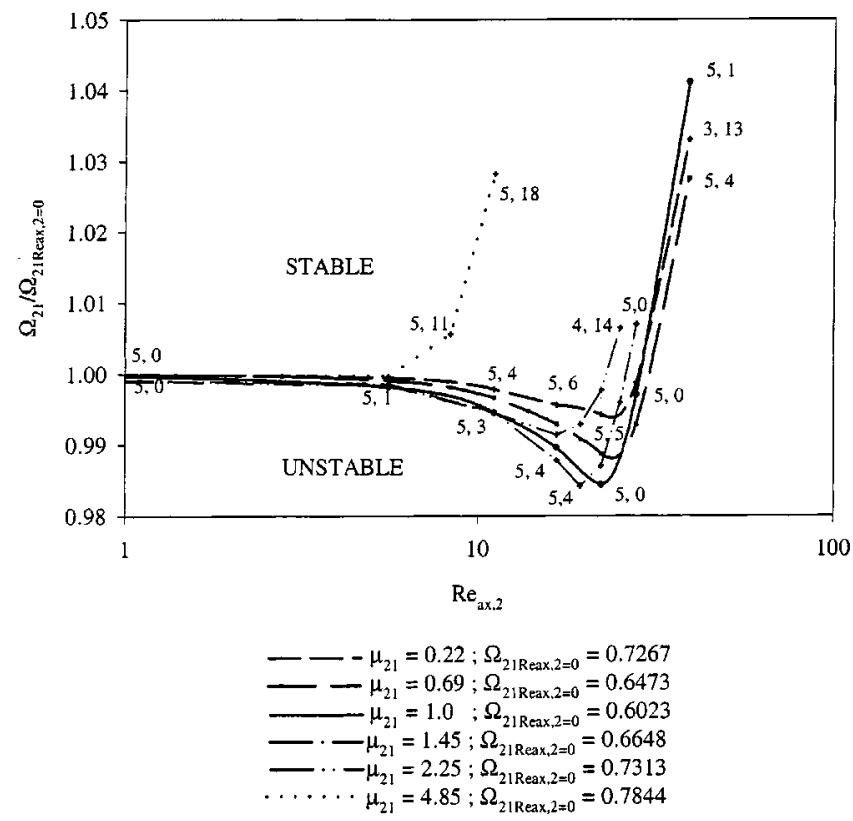

FIG. 10. The effect of viscosity ratio on the critical rotation rate with countercurrent flow. $\rho_{21}=1.35, \mathrm{Ek}_{2}=5.04 \times 10^{-3}$, and $Q_{21}=1$. The numbers represent the critical axial and azimuthal wave numbers $\alpha_{c}$ and $n_{c}$ to the nearest integer. 


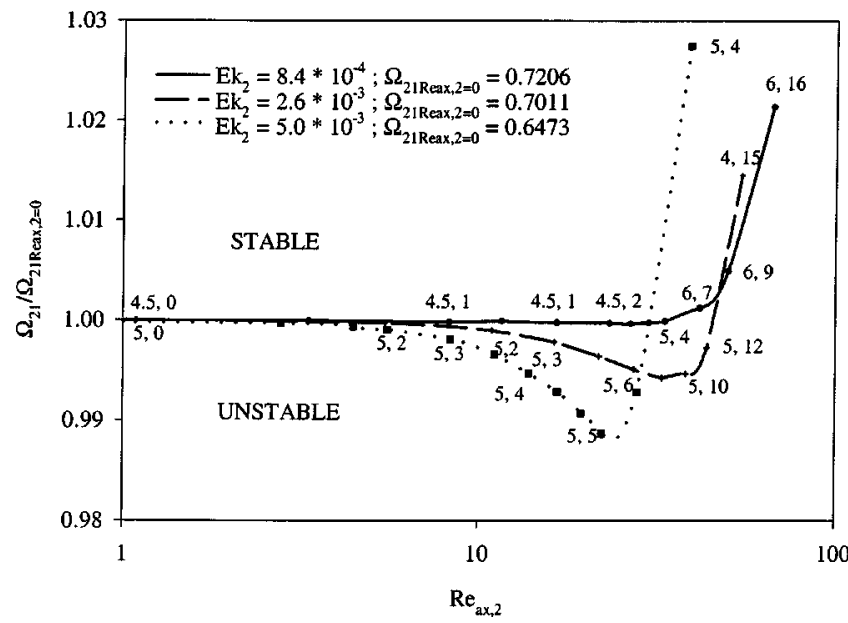

FIG. 11. The effect of viscosity on the critical rotation rate with countercurrent flow measured by axial Reynold numbers. Here $\mu_{21}=0.69, \rho_{21}$ $=1.35$, and $Q_{21}=1$. The numbers represent the critical axial and azimuthal wave numbers $\alpha_{c}$ and $n_{c}$ to the nearest integer.

scaled by viscosity). The flow is also more axisymmetric (i.e., $n_{c}$ is smaller) with higher viscosity fluids.

Finally, Fig. 13 shows that surface tension suppresses the Kelvin-Helmholtz instability as expected from other studies. ${ }^{22,24}$ Also apparent from the values in the figure legend is the very slight stabilizing effect of surface tension in the absence of counterflow: as $J^{-1}$ increases, $\Omega_{21, c}$ decreases very slightly.

\section{CONCLUSIONS}

In this paper, our previous stability analysis for two-fluid Taylor-Couette flow is extended to include pressure-driven countercurrent axial flow. As in the case without axial flow, a row of counterrotating vortices develops in each phase. Except in the case of one very thin layer or a very small gap, the vortex motion is stronger in the less viscous phase, as predicted by a generalized Rayleigh criterion. At low coun-

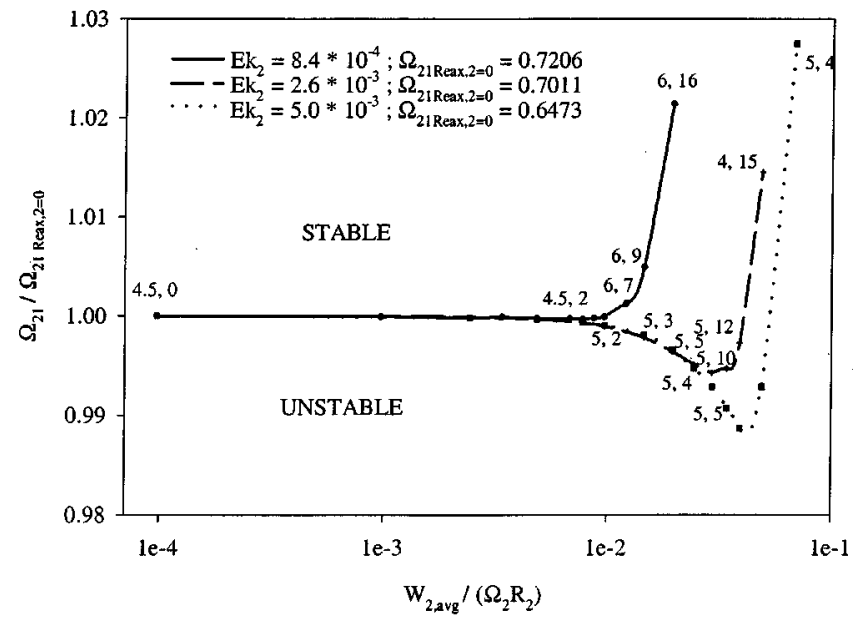

FIG. 12. The effect of viscosity on the critical rotation rate with countercurrent flow as measured by scaled axial velocity. Here $\mu_{21}=0.69, \rho_{21}$ $=1.35$, and $Q_{21}=1$. The numbers represent the critical axial and azimuthal wave numbers $\alpha_{c}$ and $n_{c}$ to the nearest integer.

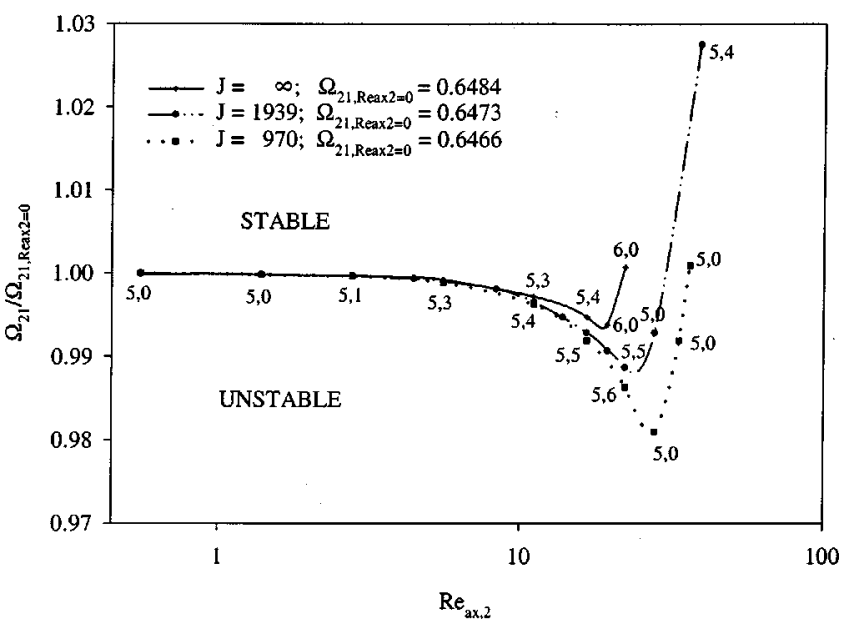

FIG. 13. The effect of surface tension on the onset of the Kelvin-Helmholtz instability. Here $\mu_{21}=0.69, \rho_{21}=1.35$, and $Q_{21}=1$. The numbers represent the critical axial and azimuthal wave numbers $\alpha_{c}$ and $n_{c}$ to the nearest integer.

tercurrent flow rates, the axial flow is stabilizing and the pattern is axisymmetric; at higher rates both the axial and azimuthal wave numbers increase. At higher countercurrent flow rates a Kelvin-Helmholtz instability appears, preceding the Taylor-Couette instability.

\section{ACKNOWLEDGMENTS}

We are indebted to E. N. Lightfoot, who first suggested that the Taylor-Couette instability might be useful for liquidliquid extraction. This work was supported by NSF CAREER award CTS-9502677 and a Shell Faculty Fellowship.

${ }^{1}$ G. I. Taylor, "Stability of a viscous liquid contained between two rotating cylinders," Philos. Trans. R. Soc. London, Ser. A 223, 289 (1923).

${ }^{2}$ G. Baier and M. D. Graham, "Two-fluid Taylor-Couette flow: Experiments and linear theory for immiscible liquids between corotating cylinders," Phys. Fluids 10, 3045 (1998).

${ }^{3}$ S. Cohen and D. M. Marom, "Experimental and theoretical study of a rotating annular flow reactor,' Chem. Eng. J. 27, 87 (1983).

${ }^{4}$ U. B. Holeschovsky and C. L. Cooney, "Quantitative description of ultrafiltration in a rotational filtration device," AIChE. J. 37, 1219 (1991).

${ }^{5}$ G. Iosilevskii, H. Brenner, C. M. V. Moore, and C. L. Cooney, "Mass transport and chemical reaction in Taylor vortex flows with entrained catalyst particles: applications to a novel class of immobilized enzyme biochemical reactors," Philos. Trans. R. Soc. London, Ser. A 345, 259 (1993).

${ }^{6}$ D. A. Janes, N. H. Thomas, and J. A. Callow, " Demonstration of a bubble free annular vortex membrane bioreactor for batch culture of red beet cells," Biotechnol. Tech. 1, 257 (1987).

${ }^{7}$ K. H. Kroner, V. Nissinen, and H. Ziegler, "Improved dynamic filtration of microbial suspensions," Bio/Technology 5, 921 (1987).

${ }^{8} \mathrm{~K}$. H. Kroner and V. Nissinen, "Dynamic filtration of microbial suspensions using and axially rotating filter,' J. Membr. Sci. 36, 85 (1988).

${ }^{9}$ T. Murase, E. Iritani, P. Chidphong, K. Kano, K. Atsumi, and M. Shirato, "High-speed microfiltration using a rotating cylindrical ceramic membrane," Int. Chem. Eng. 31, 370 (1991).

${ }^{10}$ J. G. Schechowskii, C. A. Koval, and R. D. Noble, "A Taylor vortex reactor for heterogeneous photocatalysis," Chem. Eng. Sci. 50, 3163 (1995).

${ }^{11} \mathrm{H}$. Winzeler and G. Belfort, "Enhanced performance for pressure-driven membrane processes: the argument for fluid instabilities," J. Membr. Sci. 80, 35 (1993).

${ }^{12}$ N. L. Abbott and T. A. Hatton, "Liquid-liquid extraction for protein separations,'” Chem. Eng. Prog. Aug, 31 (1988). 
${ }^{13}$ G. Baier, M. D. Graham, and E. N. Lightfoot, "Mass transport in a novel two-fluid Taylor vortex extractor,', to appear in AIChE. J.

${ }^{14}$ D. D. Joseph, Y. Renardy, M. Renardy, and K. Nguyen, "Stability of rigid motions and rollers in bicomponent flows of immiscible liquids,' J. Fluid Mech. 153, 151 (1985).

${ }^{15}$ Y. Renardy and D. D. Joseph, "Couette flow of two fluids between concentric cylinders,', J. Fluid Mech. 150, 381 (1985).

${ }^{16}$ D. D. Joseph, K. Nguyen, and G. S. Beavers, "Non-uniqueness and stability of the configuration of flow of immiscible fluids with different viscosities,', J. Fluid Mech. 141, 319 (1984).

${ }^{17}$ D. D. Joseph, and Y. Y. Renardy, Fundamentals of Two-Fluid Dynamics. Part 1: Mathematical Theory and Applications (Springer-Verlag, New York, 1993).

${ }^{18}$ R. J. Campero and R. D. Vigil, "Flow patterns in liquid-liquid TaylorCouette-Poiseuille flow,' Ind. Eng. Chem. Res. 38, 1094 (1999).

${ }^{19}$ Y. Toya and I. Nakamura, "Instability of two-fluid Taylor vortex flow,", Trans. Jpn. Soc. Mech. Eng., Ser. B 63, 35 (1997).

${ }^{20}$ C. S. Yih, "Instability due to viscosity stratification,'” J. Fluid Mech. 27, 337 (1962)

${ }^{21}$ C. T. Gallagher, D. T. Leighton, and M. J. McCready, "Experimental investigation of a two-layer shearing instability in a cylindrical Couette cell,', Phys. Fluids 8, 2385 (1996).

${ }^{22}$ P. G. Drazin and W. H. Reid, Hydrodynamic Stability (Cambridge U.P., Cambridge, 1981).

${ }^{23}$ A. E. Hosoi and L. Mahadevan, "Axial instability of a free-surface front in a partially filled horizontal rotating cylinder,' Phys. Fluids 11, 97 (1999).

${ }^{24}$ S. Chandrasekhar, Hydrodynamic and Hydromagnetic Stability (Dover, New York, 1981).

${ }^{25}$ B. S. Ng and E. R. Turner, "On the linear stability of spiral flow between rotating cylinders,' Proc. R. Soc. London, Ser. A 382, 83 (1982).

${ }^{26} \mathrm{~T}$. Howes and M. Rudman, "Flow and axial dispersion simulation for travelling axisymmetric Taylor vortices,'” AIChE. J. 44, 255 (1998).

${ }^{27}$ R. C. Giordano, R. L. C. Giordano, D. M. F. Prazeres, and C. L. Cooney,
"Analysis of a Taylor-Poiseuille vortex flow reactor- I: Flow patterns and mass transfer characteristics,'” Chem. Eng. Sci. 53, 3635 (1998).

${ }^{28}$ D. I. Takeuchi and D. F. Jankowski, "A numerical and experimental investigation of the stability of spiral Poiseuille flow,' J. Fluid Mech. 102, 101 (1981).

${ }^{29}$ H. A. Snyder, "Experiments on the stability of spiral flow at low axial Reynolds numbers,' Proc. R. Soc. London, Ser. A 265, 198 (1962).

${ }^{30}$ R. J. Donnelly and D. Fultz, "Experiments on the stability of spiral flow between corotating cylinders," Proc. Natl. Acad. Sci. USA 46, 1150 (1960).

${ }^{31}$ R. M. Lueptow, A. Docter, and K. Min, "Stability of axial flow in an annulus with a rotating inner cylinder,' Phys. Fluids A 4, 2446 (1992).

${ }^{32}$ A. Y. Weisberg, I. G. Kevrekidis, and A. J. Smits, "Delaying transition in Taylor-Couette flow with axial motion of the inner cylinder,' J. Fluid Mech. 348, 141 (1997).

${ }^{33}$ F. Marques and J. M. Lopez, "Taylor-Couette flow with axial oscillations of the inner cylinder: Floquet analysis of the basic flow,' J. Fluid Mech. 348, 153 (1997).

${ }^{34}$ M. D. Graham, "Effect of axial flow on viscoelastic Taylor-Couette instability,', J. Fluid Mech. 360, 341 (1998).

${ }^{35}$ V. V. Ramanan, K. A. Kumar, and M. D. Graham, 'Stability of viscoelastic shear flows subjected to steady or oscillatory transverse flow,' J. Fluid Mech. 379, 255 (1999).

${ }^{36}$ C. Canuto, M. Y. Hussaini, A. Quarteroni, and T. A. Zang, Spectral Methods in Fluid Dynamics (Springer-Verlag, New York, 1988).

${ }^{37}$ B. S. Garbow, "Algorithm 535: the QZ algorithm to solve the generalized eigenvalue problem for complex matrices,' ACM Trans. Math. Softw. 4, 404 (1978).

${ }^{38}$ J. Guckenheimer and P. J. Holmes, Nonlinear Oscillations, Dynamical Systems and Bifurcations of Vector Fields (corrected second printing (Springer-Verlag, New York, 1986).

${ }^{39}$ L. G. Leal, Laminar Flow and Convective Transport Processes (Butterworth-Heinemann, Boston, 1992). 